\title{
Use of magnetic powder to effectively improve the performance of sequencing batch reactors (SBRs) in municipal wastewater treatment
}

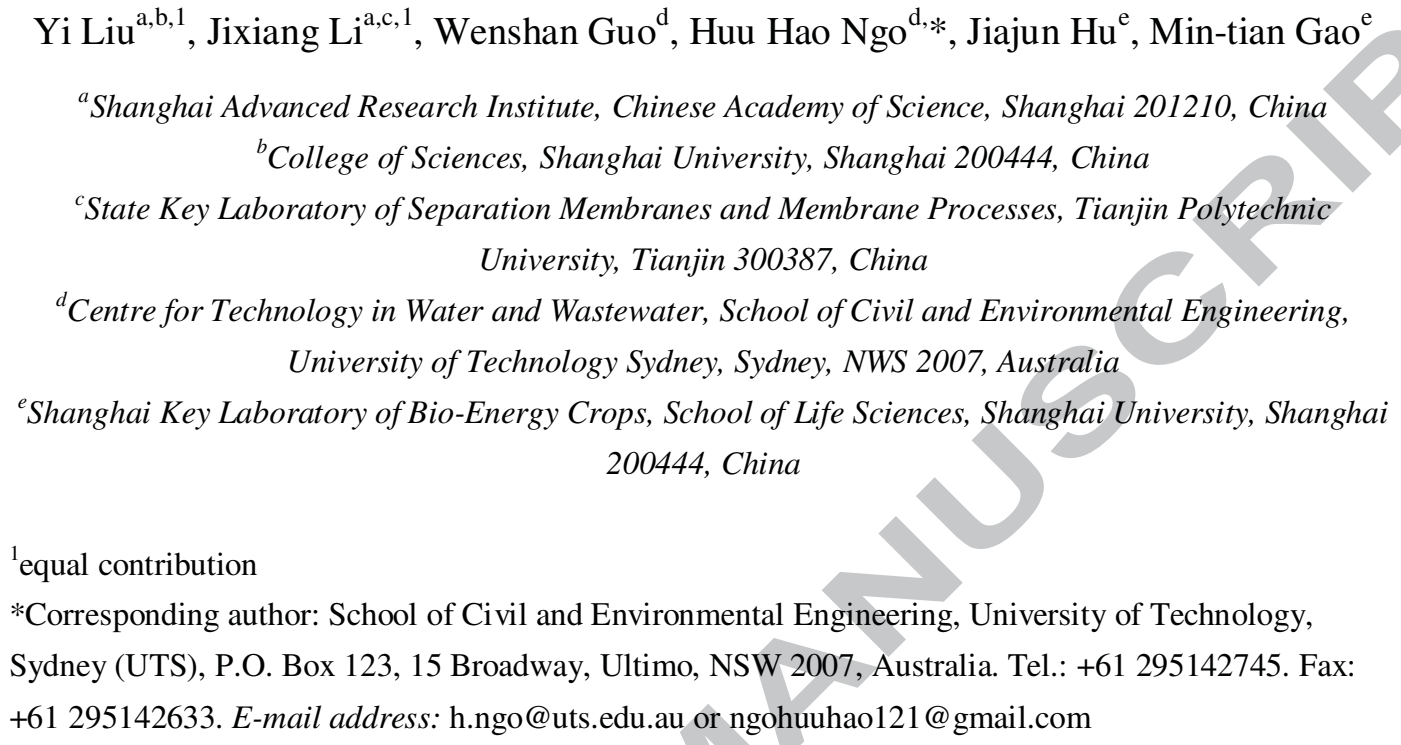

\section{Abstract}

This study aims to investigate the effect of adding magnetic powder in the sequencing batch reactor (SBR) on the reactor performance and microbial community. Results indicated that, the magnetic activated sludge sequencing batch reactor (MAS-SBR) had $7.76 \%$ and $4.76 \%$ higher ammonia nitrogen $\left(\mathrm{NH}_{4}{ }^{+}-\mathrm{N}\right)$ and chemical oxygen demand (COD) removal efficiencies than that of the conventional SBR (C-SBR). The MAS-SBR also achieved $6.86 \%$ sludge reduction compared with the C-SBR.

High-throughput sequencing demonstrated that the dominant phyla of both SBRs (present as $\geq 1 \%$ of the sequence reads) were Protebacteria, Bacteroidetes, Chloroflexi, Saccharibacteria, Chlorobi, Firmicutes, Actinobactoria, Acidobacteria, Planctomycetes and unclassified_Bacteria. The relative abundance of Protebacteria and Bacteroidetes simultaneously declined whereas the other 8 phyla increased following the addition of 
magnetic powder. Adding magnetic powder in the SBR significantly affected the microbial diversity and richness of activated sludge, consequently affecting the reactor performance.

Keywords: Magnetic activated sludge, sequencing batch reactor, sludge reduction, microbial diversity and richness, high-throughput sequencing

\section{Introduction}

Sequencing batch reactor (SBR) has been widely used in municipal and industrial wastewater treatment, as it provides several advantages such as the flexibility of process , cost effectiveness, and high biodegradation efficiency (Singh and Srivastava, 2010). However, the sludge generated in the SBR process is almost 0.3-0.5 g dry biomass produced per g soluble COD removed, and the treatment expense of excessive sludge accounts for as much $60 \%$ of the total plant operation costs (Ying et al., 2010). It should be realized currently that finding out the efficient and eco-friendly methods to minimize excess sludge production is of great importance. Recently, researchers (Nazif et al., 2016; Li et al., 2016; Raj et al., 2013) achieved successfully excess sludge reduction (20-70\%) using oxic-settling-anoxic (OSA) system, metabolic uncouplers and anaerobic/anoxic/oxic (AAO) system. However, these methods cannot avoid the drawbacks such as additional capital, operating costs, and potentially environmental impact as a part of chemical uncouplers are difficult to metabolic and will cause uncertain effects on human health eventually.
Considering its environmentally friendly and positive magnetic bio-effect, the 
magnetic activated sludge (MAS) process has been used to improve reactor

53 performance, especially sludge reduction. Ji et al. (2010) and Łebkowska et al. (2011)

54 discovered that the static magnetic field exerted a positive effect on activated sludge

55 including biomass growth, dehydrogenase activity and organic matter biodegradation.

Ma et al. (2017) reported that $10-60 \mathrm{mg} / \mathrm{L} \mathrm{Fe}_{3} \mathrm{O}_{4}$ nanoparticles improved denitrification,

57 phosphorus removal and enzymatic activity. Liu et al. (2015) compared the

conventional activated sludge (CAS) and the MAS processes for the removal of

pollutants. Results showed that adding the magnetic powder had no negative influence

on the growth of activated sludge, resulting in higher ammonia nitrogen removal

efficiency $(88.68 \pm 7.98 \%)$. Yao et al. (2013) developed a novel magnetic carrier with

surface magnetic field of $4 \mathrm{mT}$ to study the magnetic enhanced bio-effect on

nitrification in sequencing batch biofilm reactors. They revealed that nitrite and

ammonia oxidation activities in biofilm were promoted in the magnetic carrier reactor

compared with non-magnetic carrier reactor. Ying et al. (2010) also obtained an average

of $91 \%$ COD removal efficiency when using the MAS process. Furthermore, they found

67 that the MAS process could possibly maintain high MLVSS concentration and lower

the sludge loading rate, and consequently resulted in low sludge production. Zhou et al.

69 (2015) developed a kind of modified $\mathrm{Fe}_{3} \mathrm{O}_{4}$ nanoparticles as an efficient biomimic

70 catalyst for promoting activated sludge anaerobic hydrolysis,, Their results indicated

that sludge reduction efficiency increased from an initial $17.0 \%$ to $35.3 \%$ under optimal

conditions. 
To the best of our knowledge, the biodegradation process depends on the microorganisms in the bioreactor, thus we should pay more attention to the microbial communities involved for providing insight into how microorganisms influence reactor performance. Especially, with the rapid development of modern molecular biological methods, such as high-throughput sequencing, it is easier to analyze the complex microbial communities. (Neoh et al., 2017; Zhang et al., 2016; Yuan et al., 2016).

This study aims to evaluate the use of magnetic powder for enhancing pollutants removal as well as reducing sludge production in the SBR. Further, high-throughput sequencing technology served to analyze the microbial community and composition in the SBR, in order to reveal the impact of added magnetic powder on the microbial communities and how to improve the performance of SBR.

\section{Materials and methods}

\subsection{SBR and operating conditions}

Two SBRs used in this study consisted of two aerobic reactors (each having an effective volume of $4 \mathrm{~L}$ ). One was a MAS sequencing batch reactor (MAS-SBR) with 1g/L added magnetic powder $(9.119 \mu \mathrm{m}$, Sinopharm Chemical Regent Co., Ltd, China), and the other was a conventional SBR (C-SBR) without adding magnetic powder. Synthetic wastewater was used as feed to both SBRs. The reactors were seeded with aerobic activated sludge from a local municipal wastewater plant (Bailonggang Wastewater Treatment Plant, Shanghai, China), and operated under the same conditions for 60 days. The aeration intensity was set at $0.75 \mathrm{~m}^{3} / \mathrm{h}$ and the hydraulic retention time 
94 (HRT) was $8 \mathrm{~h}$ with extra $1 \mathrm{~h}$ setting time. No sludge waste occurred throughout 60

95 days from both reactors, except samples taken to measure the suspended solids. The

96 experiment temperature was conducted at $28-32{ }^{\circ} \mathrm{C}$.

\subsection{Raw wastewater and physiochemical analysis}

98 Raw wastewater was prepared every day referring to Liu et al. (2005). Chemical oxygen demand (COD), ammonia-nitrogen $\left(\mathrm{NH}_{4}{ }^{+}-\mathrm{N}\right)$ and mixed volatile suspended solids (MLSS) were measured according to Chinese NEPA standard methods (2002).

The influent COD were $290.1 \pm 7.4 \mathrm{mg} / \mathrm{L}$ and $287 \pm 4.57 \mathrm{mg} / \mathrm{L}$ for the C-SBR and

MAS-SBR, respectively. The influent $\mathrm{NH}_{4}{ }^{+}-\mathrm{N}$ were $65.7 \pm 2.7 \mathrm{mg} / \mathrm{L}$ and $64.6 \pm 2.15$ $\mathrm{mg} / \mathrm{L}$ for the C-SBR and the MAS-SBR, respectively. The particle sizes of magnetic powder and sludge samples were measured using a static light scattering particle size sludge activity was measured by $\mathrm{pH}$ value method whose principle is the concentration of $\mathrm{CO}_{2}$ accumulated by cellular respiration (Li et al., 2007) analyzer (Malvern 2000, Britain). The measurement of sludge volume after $30 \mathrm{~min}$ of settling $\left(\mathrm{SVI}_{30}\right)$ was used for describing sedimentation performance of sludge. The

\subsection{Microbial community and diversity analysis}

\subsubsection{DNA extraction and PCR amplification}

111 To comprehensively analyze the microbial communities in the SBRs, 2 mixed liquor

112 samples (one sample for each) were collected from the C-SBR and the MAS-SBR at the

113 end of the experiments for Illumina MiSeq sequence analysis. Microbial DNA was

114 extracted using the E.Z.N.A.® Soil DNA Kit (Omega Bio-tek, Norcross, GA, U.S.) 
115

116

according to manufacturer's protocols.

\subsubsection{4 sequencing and phylogenetic classification}

Amplicons were extracted, purified and quantified according to the manufacturer's instructions. Purified amplicons were pooled in equimolar and paired-end sequenced (2 $\times 250$ ) on an Illumina MiSeq platform. The raw reads were deposited into the NCBI

Sequence Read Archive (SRA) database (Accession Number: SRP101681). Operational Units (OTUs) were clustered with 97\% similarity using UPARSE, and chimeric sequences were identified and removed using UCHIME. The taxonomy of each $16 \mathrm{~S}$ rRNA gene sequence was analyzed by RDP Classifier against the silva (SSU123)16S rRNA database using the confidence threshold of $70 \%$. According to the rarefaction curves, Shannon and Chao indices were calculated to compare the microbial diversity and richness between the C-SBR and the MAS-SBR samples.

\section{Results and Discussion}

\subsection{The performance of the SBRs}

After nearly one month acclimatization, both SBRs successfully reached steady state.

The COD and $\mathrm{NH}_{4}{ }^{+}-\mathrm{N}$ removals are provided in Figure 1. It can be seen from Figure 1

that adding magnetic powder in the SBR had no negative impact on the removals of COD and $\mathrm{NH}_{4}{ }^{+}-\mathrm{N}$. The average $\mathrm{COD}$ and $\mathrm{NH}_{4}{ }^{+}-\mathrm{N}$ removal efficiencies increased from $83.60 \pm 11.02 \%$ to $88.36 \pm 7.43 \%, 78.54 \pm 18.59 \%$ to $86.3 \pm 13.13 \%$, respectively. Thus, adding magnetic powder in the SBR successfully enhance the removal of the pollutants.

\subsection{Activated sludge properties}


136 At the beginning of the experiments, the MLSS concentrations of both SBRs were

$1373.146 \pm 0.055 \mathrm{~g} / \mathrm{L}$. By the end, the MLSS concentrations of the C-SBR and the

138 MAS-SBR reached up to 5.376 $\pm 0.067 \mathrm{~g} / \mathrm{L}$ and $5.160 \pm 0.061 \mathrm{~g} / \mathrm{L}$ respectively. The

139 amount of sludge increased by $70.88 \%$ in the C-SBR and $64.02 \%$ in the MAS-SBR,

140 suggesting that $6.86 \%$ sludge reduction was obtained by adding magnetic powder in the

141 SBR. At the same time the $\mathrm{SVI}_{30}$ tests demonstrated that adding magnetic powder could

142 improve the sedimentation property of sludge. In other words, the possibility of sludge

143 bulking can be partly decreased by adding magnetic powder. Meanwhile, according to

144 the $\mathrm{pH}$ value method testing, the sludge activity measured as $\Delta\left[\mathrm{CO}_{2}\right]$ increased from

$1450.088 \mu \mathrm{mol} / \mathrm{g} \cdot \mathrm{min}$ of the C-SBR to $0.119 \mu \mathrm{mol} / \mathrm{g} \cdot \mathrm{min}$ of the MAS-SBR, indicating that

146 adding magnetic powder in the SBR had a positive effect on sludge activity. The

147 average particle size distribution size was $40.821 \mu \mathrm{m}$ for the C-SBR and $37.765 \mu \mathrm{m}$ for

148 the MAS-SBR. Thus, adding magnetic powder had little bioflocculation effect on

149 activated sludge. It can be inferred that magnetic bio-effect may affect the microbial

150 communities in the SBRs, and further improved the reactor performance. Thus it is

151 necessary to further analyze the diversity, composition and dominant species of

152 microbial communities in the SBRs.

153 3.3 Microbial community and diversity analysis

$154 \quad$ 3.3.1 Overall analysis of amplicon sequencing

155 The microbial community and composition of activated sludge samples in the SBRs

156 were investigated through high-throughput sequencing. After necessary quality filtering, 
157

the valid sequences of activated sludge samples in the C-SBR and the MAS-SBR were 32454 and 37494, respectively. Alignment and clustering of the sequences showed that the OTUs based on a 97\% sequence identity of the C-SBR and the MAS-SBR were 492 and 493, respectively. In summary, the microbial communities in the SBRs consist of 30 phyla, 58 classes, 95 orders, 156 families, 223 genera, 344 species (relative abundance $\geq 0.1 \%$ ). The rarefaction curves approached the plateau phase at 400 reads for both samples, suggesting that the sequencing depth was sufficient to capture most of the microbial diversity. Also, the Good's coverage of the sludge samples in both SBRs were more than 0.998 , indicating that the sequence library obtained from each sludge sample could cover the microbial diversity of the SBR. Moreover, Shannon indices showed that the MAS-SBR (4.8) contained higher level of community diversity than the C-SBR (4.59) whereas Chao indices confirmed the opposite result in species richness.

\subsubsection{Taxonomic classification of the microbial communities}

To better understand the microbial communities of the C-SBR and the MAS-SBR systems, the sequence reads were taxonomically classified and the relative abundance was calculated at the phylum and genus level for each sample. The results are visualized

in Figure 2 and Table 1. At the phylum level, the top 10 dominant phyla present as $\geq 1 \%$ of the sequence reads in the C-SBR and the MAS-SBR were similar, including Proteobacteria (33.9\% and 28.1\%), Bacteroidetes (32.7\% and 26.0\%), Chloroflexi (11.9\% and 18.6\%), Saccharibateria (5.0\% and 5.1\%), Chlorobi (4.2\% and 5.4\%), Firmicutes (4.2\% and 4.5\%), Actinobactoria (2.95\% and 3.85\%), Acidobacteria $(2.05 \%$ 
and $3.58 \%)$, Planctomycetes (1.02\% and $1.43 \%)$ and unclassified_Bacteria (1.11\% and $1.45 \%)$, respectively. It can be seen that the relative abundance of Protebacteria and Bacteroidetes simultaneously declined from $33.9 \%$ and $32.7 \%$ to $28.1 \%$ and $26.0 \%$, respectively, whereas the other phyla increased by $6.7 \%, 0.1 \%, 1.2 \%, 0.3 \%, 0.9 \%, 0.8 \%$, $0.41 \%$ and $0.34 \%$, respectively. It is reported that Bacteroidetes play a critical role in degradation of polysaccharides and starch which are considered to be COD in wastewater (Shu et al., 2015). Although the relative abundance of Bacteriodetes declined by $6.7 \%$ with added magnetic powder, there is no negative effect on COD removal efficiency, suggesting functional-sosurplus Bacteriodetes existed in both SBRs. Chloroflexi have been identified as the major participants in the biological treatment in digesting long chain fermenting sugars into short chain fatty acids precursors (Yamada and Sekiguchi, 2009). Herein, the increased abundance of Chloroflexi in the MAS-SBR may provide evidence for improved COD removal. At the genus level, the relative abundance of Nitrosomonas fell from $0.017 \%$ in the C-SBR to $0.004 \%$ in the MAS-MBR, whereas the relative abundance of Nitrosomonadaceae, Nitrospira and Candidatus_Nitrotoga increased from $0.665 \%, 0.065 \%$ and $0.017 \%$ in the C-SBR to $0.743 \%, 0.128 \%$ and $0.021 \%$ in the MAS-MBR, respectively. Nitrosomonas and Nitrosomonadaceae are related to the process of ammonia oxidization to nitrite, and Nitrospira and Candidatus_Nitrotoga are closely associated with the process of nitrite oxidation to nitrate (Ma et al., 2017). Thus, the genera variation consequently affected the processes of ammonia oxidation and nitrite oxidation in the SBR, which provided 
199

evidence of $7.76 \%$ higher $\mathrm{NH}_{4}{ }^{+}-\mathrm{N}$ removal efficiency by adding magnetic powder in the SBR. Similarly, the relative abundance of Clostridium_sensu_stricto_13 increased from $3.28 \%$ in the C-SBR to $3.94 \%$ in the MAS-SBR. It has been reported that the genera Clostridia had the ability to degrade biomass and fermentation of the wastewater (Lynd et al., 2002). Maybe it can be used to explain the $6.68 \%$ sludge reduction by the addition of magnetic powder in the SBR. Compared to the C-SBR, the relative abundances of some genera (e.g. Simplicispira, Commonadaceae, Thauera, Chryseobacterium and Saprospiaceae) decreased or increased in the MAS-SBR. Thus, adding magnetic powder in the SBR significantly affected the microbial diversity and richness of activated sludge, and consequently affected the reactor performance.

\section{Conclusion}

Adding magnetic powder in the SBR has definite potential in removal pollutants and reducing sludge production. The differences between the C-SBR and the MAS-SBR were clearly observed, such as $\mathrm{COD}$ and $\mathrm{NH}_{4}{ }^{+}-\mathrm{N}$ removals, sludge properties and dominant bacteria in activated sludge. High-throughput sequencing helped to understand the effect of adding magnetic powder on the reactor performance and microbial community in activated sludge.

More detailed information such as PCR amplification, compositions of synthetic wastewater, the $\mathrm{SV}_{30}$ results during the operation and SEM images of C-SBR and MAS-SBR can be found in Supplementary Material. 
220

221 The authors appreciate the financial support of the National Natural Science

\section{Acknowledgements} Foundation of China (No. 51408588 and No. 21307093), the Youth Innovation Promotion Association (No. 2017353), the Fund for Science and Technology

Development of Pudong (No. PKJ2014-C12), the State Key Laboratory of Separation

Membranes and Membrane Processes (Tianjin Polytechnic University, No. M2-201605),

the Scientific Research Projects of Shanghai Science and Technology Committee (No.

14540500600 and No. 16391902000). The authors are also grateful for the joint collaboration among SARI, Shanghai University and UTS.

\section{References}

1. Ji, Y.L., Wang, Y.H., Sun, J.S., Yan, T.Y., Li, J., Zhao, T.T., Yin, X.H., Sun, C.J., 2010. Enhancement of biological treatment of wastewater by magnetic field. Bioresour. Technol. 101, 8535-8540.

2. Łebkowska, M., Rutkowskanarożniak, A., Pajor, E., Pochanke, Z., 2011. Effect of a static magnetic field on formaldehyde biodegradation in wastewater by activated sludge. Bioresour. Technol. 102, 8777-8782.

3. Li, J.X., Qiao, L., Zhao, J.H., He, S.Y., Hu, Z.T., Zhang, S.L., 2007. Application of sludge activity measurement by $\mathrm{pH}$ value method in engineering. Technology of water treatment. 33 (12), 62-65.

4. Li, P., Li, H.C., Li, J., Guo, X.S., Liu, J.X., Xiao, B.Y., 2016. Evaluation of sludge reduction of three metabolic uncouplers in laboratory-scale anaerobic-anoxic-oxic 
process. Bioresour. Technol. 221, 31-36.

5. Liu, R., Huang, X., Xi, J.Y., Qian, Y., 2005. Microbial behaviour in a membrane bioreactor with complete sludge retention. Process Biochem. 40, 3165-3170.

6. Liu, Z.M., Liang, Z., Wu, S.G., Liu, F., 2015. Treatment of municipal wastewater by a magnetic activated sludge device. Desalin. Water Treat. 53, 909-918.

7. Lynd, L.R., Weimer, P.J., Zyl, W.H., Pretorius, I.S., 2002. Microbial cellulose utilization: fundamentals and biotechnology. Microbiolo. Mol. Biol. R. 66, 506-577.

8. Ma, B., Wang, S., Li, Z.W., Gao, M.C., Li, S.S., Guo, L., She, Z.L., Zhao, Y.G., Zheng, D., Jin, C.J., Wang, X.J., Gao, F., 2017. Magnetic $\mathrm{Fe}_{3} \mathrm{O}_{4}$ nanoparticles induced effects on performance and microbial community of activated sludge from a sequencing batch reactor under long-term exposure. Bioresour. Technol. 225, $377-385$.

9. Nazif, S., Mehrdadi, N., Zare, S., Mosavari, S., 2016. Improvement and prediction of OSA system performance in sludge reduction through integration with thermal and mechanical treatment. Water Sci. Technol. 74(9), 2087-2096.

10. Neoh, C.H., Yung, P.Y., Noor, Z.Z., Razak, M.H., Aris, A., Din, M.F.M., Ibrahim, Z., 2017. Correlation between microbial community structure and performances of membrane bioreactor for treatment of palm oil mill effluent. Chem. Eng. J. 308, 656-663.

11. Raj, S.E., Banu, J.R., Kaliappan, S., Yeom, I.-T., Kumar, S.A., 2013. Effects of sidestream, low temperature phosphorus recovery on the performance of 
262

263

264

anaerobic/anoxic/oxic systems integrated with sludge pretreatment. Bioresour. Technol. 140, 376-384.

12. Shu, D.T., He, Y.L., Yue, H., Wang, Q.Y., 2015. Microbial structures and community functions of anaerobic sludge in six full-scale wastewater treatment plants as revealed by 454-high-throughput pyrosequencing. Bioresour. Technol. 186, $163-172$

13. Singh, M., Srivastava, R.K., 2010. Sequencing batch reactor technology for biological wastewater treatment: a review. Asia-Pac. J. Chem. Eng. 6, 3-13.

14. Yamada and Sekiguchi, 2009. Cultivation of uncultured chloroflexi subphyla: significance and ecophysiology of formerly uncultured chloroflexi 'subphylum i' with natural and biotechnological relevance. Microbes Environ. 24(3), 205-16.

15. Yao, C., Lei, H.Y., Yu, Q., Li, S.P., Li, H.L., Chen, K., Zhang, X.H., 2013. Application of magnetic enhanced bio-effect on nitrification: a comparative study of magnetic and non-magnetic carriers. Water Sci. Technol. 67(6), 1280-1288.

16. Ying, C., Umetsu, K., Ihara, I., Sakai, Y., Yamashiro, T., 2010. Simultaneous removal of organic matter and nitrogen from milking parlor wastewater by a magnetic activated sludge (MAS) process. Bioresour. Technol. 101, 4349-4353.

17. Yuan, Y., Liu, J.J., Ma, B., Liu, Y., Wang, B., Peng, Y.Z., 2016. Improving municipal wastewater nitrogen and phosphorous removal by feeding sludge fermentation products to sequencing batch reactor (SBR). Bioresour. Technol. 222, 326-334. 
283

284

285

286

287

18. Zhang, Y., Li, B., Xu, R.X., Wang, G.X., Zhou, Y., Xie, B., 2016. Effects of pressurized aeration on organic degradation efficiency and bacterial community structure of activated sludge treating saline wastewater. Bioresour. Technol. 222, 182-189.

19. Zhou, L.F., Huang, B.B., Huang, D.W., Niu, C.G., Zeng, G.M., Ruan, M., Zhang, X.G., 2015. A feasible strategy for promoting activated sludge hydrolysis by using ironporphyrin modified $\mathrm{Fe}_{3} \mathrm{O}_{4}$ nanoparticles as an efficient biomimic catalyst. Chem. Eng. J. 280, 248-255. 
$295 \quad$ Figure Captions

296 Figure 1: Effect of adding magnetic powder in the SBR on COD and $\mathrm{NH}_{4}{ }^{+}-\mathrm{N}$ removals

297 Figure 2: Taxonomic classification of the microbial communities at phylum level in 298 both SBRs, present as $\geq 1 \%$ of the sequence reads in at least one sample.

299

300

301

302

303

304

305

306

307

308

309

310

311

312 


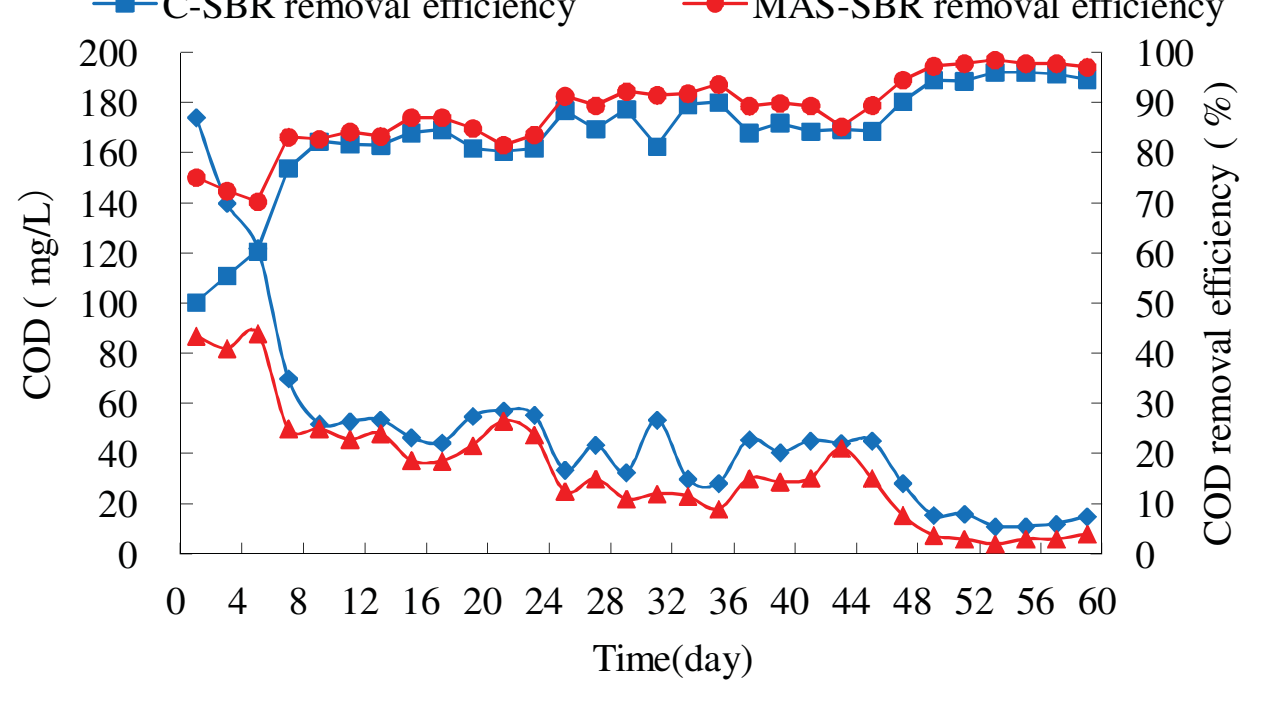

328
329
330
331
332
333
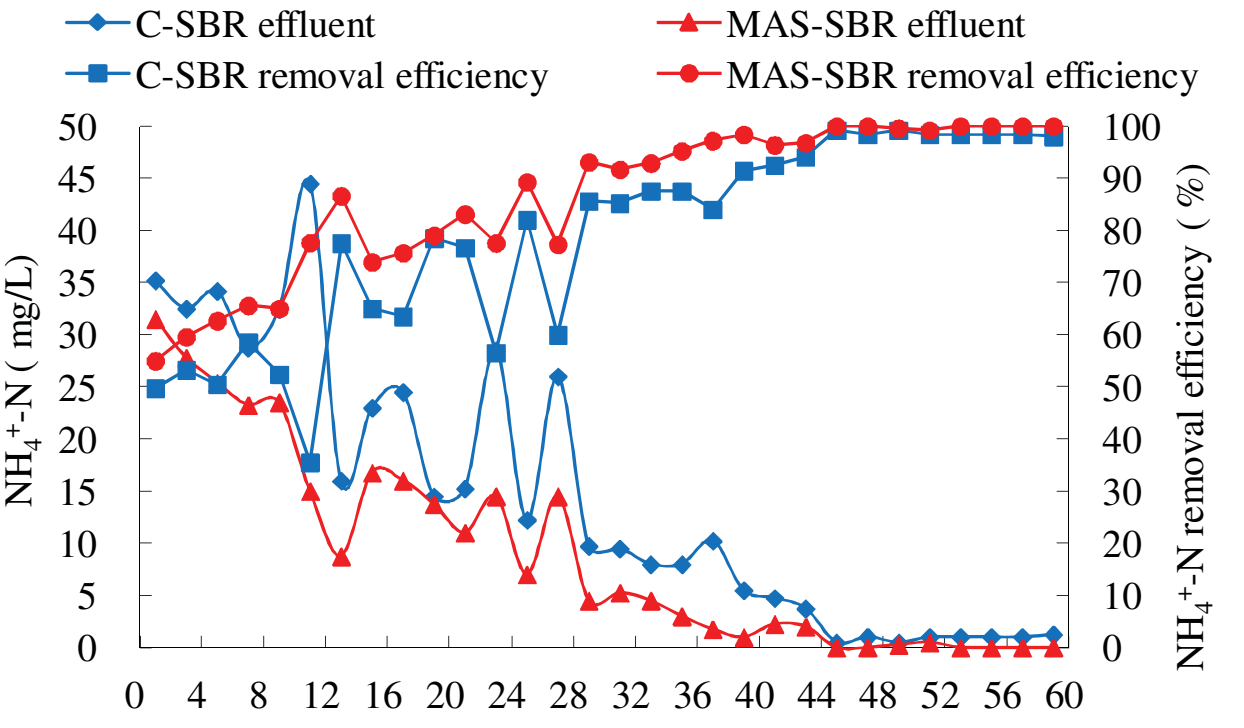

Time(day) 
$337 \quad$ Fig. 1.

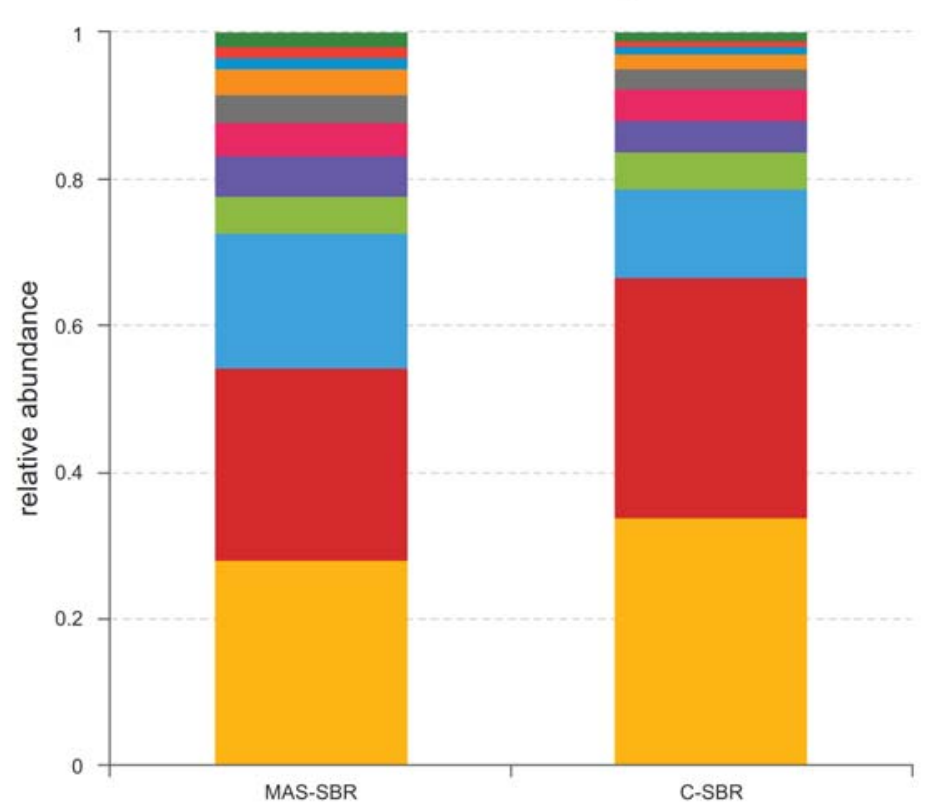

unclassified_k_norank

Planctomycetes

Acidobacteria

Actinobacteria

Firmicutes

Chlorobi

Saccharibacteria

Chloroflexi

Bacteroidetes

Proteobacteria

others

339 Fig. 2.

340

341

342

343

344

345 
Table 1

353 The comparison of the main genera in the C-SBR and the MAS-SBR

\begin{tabular}{lll}
\hline Genera & C-SBR (\%) & MAS-SBR (\%) \\
\hline Nitrosomonas & 0.017 & 0.004 \\
Nitrosomonadaceae & 0.665 & 0.743 \\
Nitrospira & 0.065 & 0.128 \\
Candidatus_Nitrotoga & 0.017 & 0.021 \\
Clostridium_sensu_stricto_13 & 3.28 & 3.94 \\
Simplicispira & 4.08 & 2.78 \\
Commonadaceae & 6.67 & 6.47 \\
Thauera & 3.30 & 1.66 \\
Chryseobacterium & 12.25 & 7.16 \\
Saprospiaceae & 8.86 & 8.14 \\
Anaerolineaceae & 5.24 & 9.02 \\
Ardenticatenia & 2.53 & 3.33 \\
Saccharibacteria & 5.16 & 5.01 \\
PHOS-HE36 & 2.07 & 2.82 \\
\hline
\end{tabular}

355 Highlights studied

359 - Adding magnetic powder could enhance the removal of pollutants and sludge $360 \quad$ reduction

- Magnetic powder addition significantly enriched the microbial diversity 